\title{
Estudo retrospectivo de surtos de doenças veiculadas por alimentos, na região nordeste e Estado do Maranhão, no período de 2007 a 2019
}

\author{
Retrospective study of outbreaks of foodborne diseases in the Northeast and State of Maranhão,
} from 2007 to 2019

Estudio retrospectivo de brotes de enfermedades de transmisión alimentaria en el noreste y Estado de Maranhão, de 2007 a 2019

Recebido: 06/01/2021 | Revisado: 08/01/2021 | Aceito: 15/01/2021 | Publicado: 18/01/2021

Jaqueline Freitas Souza

ORCID: https://orcid.org/0000-0002-3046-3212 Universidade Estadual do Maranhão, Brasil E-mail: jackllinefn@hotmail.com

Antonio Carlos Freitas Souza

ORCID: https://orcid.org/0000-0002-6921-9030 Instituto de Pesquisas Científicas e Tecnológicas do Estado do Amapá, Brasil E-mail: jr_bio2005@yahoo.com.br

Francisca Neide Costa

ORCID: https://orcid.org/0000-0002-8941-241X

Universidade Estadual do Maranhão, Brasil E-mail: francisca.cca.uema@gmail.com

\begin{abstract}
Resumo
A incidência de doenças veiculadas por alimentos cresce a cada ano no Brasil, porém estima-se que um número elevado de casos não é notificado. O objetivo deste trabalho será fazer um estudo retrospectivo da ocorrência de surtos de doenças veiculadas por alimentos, no estado do Maranhão, período de 2007 a 2019, onde foi realizado um estudo descritivo do tipo transversal, utilizando-se como base de dados o Sistema de Informação de Agravos de Notificação - SINAN. No período estudado foram identificados 48 surtos de doenças de origem alimentar no estado do Maranhão, com 2.535 doentes. Os agentes etiológicos identificados foram a Salmonella sp. (6,25\%), Escherichia coli $(4,17 \%)$, rotavírus (4,17\%), Staphylococcus aureus e coliformes, ambos com 2,08\%, porém, em $81,25 \%$ dos surtos, o agente etiológico não foi identificado. A residência foi o local de ocorrência com maior número de surtos (37,5\%), seguindo de casos dispersos no bairro (12,5\%) e restaurante/padaria $(10,42 \%)$, ainda é importante citar a ocorrência de 3 surtos (6,25\%) em hospitais/Unidade de saúde. Quanto à origem, em 47,75\% dos surtos, o alimento envolvido no surto não foi identificado e a água e alimentos mistos foram os mais comuns, com frequência de $14,58 \%$ e $12,5 \%$, respectivamente. Quanto aos critérios de confirmação, em 52,08\% dos surtos foi utilizado o critério clínicoepidemiológico, 14,58\% realizaram análises bromatológicas para confirmação e 10,42\% apenas critérios clínicos, inconclusivos e ignorados representaram 22,92\%. As diferenças na quantidade de surtos notificados no período do estudo podem decorrer de uma possível subnotificação ocasionando lacunas nos resultados encontrados.
\end{abstract}

Palavras-chave: Patógenos; Inocuidade dos alimentos; Epidemiologia.

\begin{abstract}
The incidence of foodborne diseases grows every year in Brazil, but it is estimated that a high number of cases is not reported. The objective of this work will be to carry out a retrospective study of the occurrence of outbreaks of foodborne diseases in the state of Maranhão, from 2007 to 2019, where a descriptive cross-sectional study was carried out, using as a database the Information on Notifiable Diseases - SINAN. During the study period, 48 outbreaks of foodborne diseases were identified in the state of Maranhão, with 2,535 patients. The etiologic agents identified were Salmonella sp. (6.25\%), Escherichia coli $(4.17 \%)$, rotavirus $(4.17 \%)$, Staphylococcus aureus and coliforms, both with $2.08 \%$, however, in $81.25 \%$ of outbreaks, the etiologic agent is not It was identified. The residence was the place of occurrence with the highest number of outbreaks $(37.5 \%)$, followed by cases dispersed in the neighborhood (12.5\%) and restaurant / bakery $(10.42 \%)$, it is still important to mention the occurrence of 3 outbreaks $(6.25 \%)$ in hospitals / health units. As for the origin, in $47.75 \%$ of the outbreaks, the food involved in the outbreak was not identified and water and mixed foods were the most common, with a frequency of $14.58 \%$ and $12.5 \%$, respectively. As for the confirmation criteria, in $52.08 \%$ of the outbreaks the clinical-epidemiological criterion was used, $14.58 \%$ performed bromatological analyzes for confirmation and $10.42 \%$ only clinical, inconclusive and ignored criteria represented
\end{abstract}


22.92\%. Differences in the number of outbreaks reported during the study period can result from a possible underreporting, causing gaps in the results found.

Keywords: Pathogens; Food safety; Epidemiology.

\begin{abstract}
Resumen
La incidencia de enfermedades transmitidas por alimentos crece cada año en Brasil, pero se estima que no se reporta un alto número de casos. El objetivo de este trabajo será realizar un estudio retrospectivo de la ocurrencia de brotes de enfermedades transmitidas por alimentos en el estado de Maranhão, de 2007 a 2019, donde se realizó un estudio descriptivo transversal, utilizando como base de datos la Información sobre Enfermedades Notificables - SINAN. Durante el período de estudio, se identificaron 48 brotes de enfermedades de transmisión alimentaria en el estado de Maranhão, con 2.535 pacientes. Los agentes etiológicos identificados fueron Salmonella sp. (6,25\%), Escherichia coli $(4,17 \%)$, rotavirus $(4,17 \%)$, Staphylococcus aureus y coliformes, ambos con 2,08\%, sin embargo, en 81,25\% de los brotes, el agente etiológico no es Fue identificado. La residencia fue el lugar de ocurrencia con mayor número de brotes (37.5\%), seguido de los casos dispersos en el barrio (12.5\%) y restaurante / panadería (10.42\%), aún es importante mencionar la ocurrencia de 3 brotes $(6,25 \%)$ en hospitales / unidades de salud. En cuanto al origen, en el $47,75 \%$ de los brotes no se identificó el alimento involucrado en el brote y el agua y los alimentos mixtos fueron los más comunes, con una frecuencia de $14,58 \%$ y 12,5\%, respectivamente. En cuanto a los criterios de confirmación, en el 52,08\% de los brotes se utilizó el criterio clínico-epidemiológico, el 14,58\% realizó análisis bromatológicos para confirmación y el $10,42 \%$ solo los criterios clínicos, no concluyentes e ignorados representaron el 22,92\%. Las diferencias en el número de brotes notificados durante el período de estudio pueden deberse a un posible subregistro, lo que genera brechas en los resultados encontrados.
\end{abstract}

Palabras clave: Patógenos; Inocuidad de los alimentos; Epidemiología.

\title{
1. Introdução
}

Segundo a World Health Organization, estima-se que cerca de 600 milhões de pessoas sejam acometidas por doenças veiculadas por alimentos (DTA), e em torno de 420 mil mortes por ano, e acredita-se que este número vem aumentando no decorrer dos anos. São diversos os fatores que vem influenciando o surgimento de surtos de DTA, entre eles, destaca-se o processo de urbanização desordenado, a necessidade de produzir alimentos em grande escala, o progressivo aumento da população e a presença de grupos populacionais mais expostos ou vulneráveis (Brasil, 2010; Who, 2019).

No Brasil, de 2007 a 2018, foram notificados em média 686 surtos alimentares por ano, com mais de 240 mil doentes segundo os dados do Sistema de Informação de Agravos de Notificação-SINAN (Brasil, 2018). Porém, como consequência da fragilidade do Sistema Nacional de Vigilância Epidemiológica das Doenças Transmitidas por Alimentos (VE-DTA) e dos programas de controle, estas informações disponibilizadas não representam a magnitude real deste problema.

Sendo reconhecidas como um dos principais problemas de saúde pública do país, o conhecimento da causa das DTA, do número de casos da doença, hospitalizações e óbitos, são informações importantes para estabelecer estratégias e prioridades de vigilância, controle e precaução (Correia et al., 2019). Além dos impactos na saúde, a economia do país também sofre consequências com o crescimento destes índices, através da redução da renda dos indivíduos afetados, ausência nos postos de trabalho, diminuição da produtividade, gastos com cuidados médicos e com investigações de surtos, além do fechamento de negócios e a redução no consumo de produtos envolvidos, prejudicando o turismo e o comércio (Melo et al., 2018).

As regiões sudeste e sul sempre se destacaram com os altos números de surtos relatados no país, resultante da concentração populacional e de um sistema mais atuante de fiscalização e notificação. Por outro lado, a região nordeste representa apenas 15,5\% dos surtos notificados no Brasil, porém, presume-se que muitos casos não são notificados, especialmente pelas características regionais e o baixo índice de desenvolvimento humano da região (devido aos hábitos culturais de alimentação das populações de baixa renda) (Guilherme \& Esteves, 2017).

Diante disso, o objetivo deste trabalho foi fazer um estudo retrospectivo, 2007 a 2019, da ocorrência de surtos das doenças veiculadas por alimentos no estado do Maranhão, Brasil. 


\section{Metodologia}

Trata-se de uma pesquisa documental a partir de um estudo transversal retrospectivo, de natureza quantitativa (Pereira et al., 2018), onde foi analisada a ocorrência de DTA's no estado do Maranhão, no período de 2007 a 2019.

O objeto do estudo foi composto por todos os surtos de DTA's ocorridos no estado do Maranhão, notificados e registrados pelo SINAN. Os dados foram coletados por meio de relatórios disponibilizados na internet, utilizando as variáveis: número de surtos de DTA por ano de notificação, local de ocorrência, agente etiológico e alimentos envolvidos no surto, número total de doentes, número de óbitos e critérios de confirmação. A obtenção dos dados ocorreu durante os meses de julho a agosto de 2020 e o processamento dos dados foi executado em planilha, usando o programa Microsoft Office Excel® 2010, além de estatística descritiva simples.

\section{Resultados e Discussão}

Pela análise dos dados obtidos, no Maranhão, no período de 2007 a 2019 foram relatados um total de 48 surtos de doenças veiculadas por alimentos. Em 2008 ocorreu o maior número de notificações (n=11), seguido dos anos 2013 e 2012 com $(n=8)$ e $(n=5)$, respectivamente, apesar da sua extensão, o estado representa apenas $2,42 \%$ dos surtos relatados na região Nordeste (Tabela 1).

Ainda segundo os dados do SINAN, o Nordeste representa 15,5\% dos surtos relatados no Brasil, onde as regiões Sul $(33,9 \%)$ e Sudeste $(39,2 \%)$ sempre se destacaram com os índices mais elevados de notificação de doenças veiculadas por alimentos (Brasil, 2018). No entanto, muitos estados da região Nordeste apresentam número subestimados de surtos alimentares, não demonstrando a real magnitude deste problema (Guilherme \& Esteves, 2017).

Tabela 1. Surtos de doenças veiculadas por alimentos no Nordeste, com ênfase ao estado do Maranhão no período de 2007 a 2019.

\begin{tabular}{|c|c|c|c|c|c|c|c|c|c|c|c|c|c|c|c|}
\hline & & 2007 & 2008 & 2009 & 2010 & 2011 & 2012 & 2013 & 2014 & 2015 & 2016 & 2017 & 2018 & 2019 & TOTAL \\
\hline \multirow{3}{*}{ 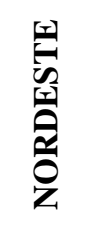 } & Surtos & 68 & 67 & 72 & 114 & 164 & 147 & 226 & 249 & 130 & 150 & 208 & 189 & 201 & 1985 \\
\hline & NTD & 2201 & 1457 & 992 & 2140 & 4476 & 2584 & 7479 & 5718 & 1806 & 3068 & 3121 & 2000 & 2315 & 39357 \\
\hline & NO & 8 & 8 & 0 & 0 & 2 & 2 & 6 & 5 & 0 & 2 & 3 & 1 & 3 & 40 \\
\hline \multirow{3}{*}{$\mathbb{z}$} & Surtos & 5 & 11 & 1 & 7 & 0 & 5 & 8 & 3 & 1 & 1 & 3 & 1 & 2 & 48 \\
\hline & NTD & 1046 & 741 & 2 & 190 & 0 & 167 & 147 & 134 & 8 & 2 & 74 & 9 & 15 & 2535 \\
\hline & NO & 8 & 7 & 0 & 0 & 0 & 0 & 0 & 0 & 0 & 0 & 0 & 0 & 0 & 15 \\
\hline \multirow{8}{*}{ 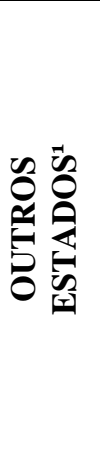 } & Alagoas & 4 & 2 & 5 & 7 & 4 & 2 & 5 & 4 & 4 & 9 & 8 & 1 & 3 & 58 \\
\hline & Bahia & 2 & 3 & 4 & 13 & 20 & 35 & 23 & 13 & 8 & 6 & 15 & 4 & 13 & 159 \\
\hline & Ceará & 15 & 9 & 16 & 17 & 41 & 24 & 32 & 26 & 7 & 29 & 26 & 25 & 22 & 289 \\
\hline & Paraíba & 1 & 3 & 6 & 5 & 6 & 1 & 5 & 5 & 3 & 1 & 1 & 1 & 2 & 40 \\
\hline & Pernambuco & 29 & 35 & 29 & 28 & 65 & 53 & 106 & 168 & 77 & 74 & 123 & 122 & 120 & 1029 \\
\hline & Piauí & 0 & 1 & 6 & 4 & 0 & 6 & 9 & 7 & 8 & 23 & 3 & 9 & 14 & 90 \\
\hline & $\begin{array}{l}\text { Rio Grande } \\
\text { do Norte }\end{array}$ & 5 & 2 & 2 & 20 & 10 & 16 & 23 & 14 & 16 & 5 & 18 & 20 & 19 & 170 \\
\hline & Sergipe & 7 & 1 & 3 & 13 & 18 & 5 & 15 & 9 & 6 & 2 & 11 & 6 & 6 & 102 \\
\hline
\end{tabular}

NTD: Número Total de Doentes; NO: Número de Óbitos; ${ }^{1}$ Número de surtos. Fonte: Autores. 
Na região nordeste, o estado mais representativo em notificação de números de surtos é Pernambuco, que notificou 1029 surtos no período de 2007 a 2019, representando 51,8\% dos surtos relatados na região Nordeste. Por este motivo, estimase que um número elevado de casos não é notificado no Maranhão, especialmente porque muitas destas enfermidades causam sintomas brandos onde o paciente não procura auxílio médico, optando pela automedicação (Sirtoli \& Comarella, 2018).

Foram registrados 2.535 casos de DTA no período estudado no Maranhão, com média de 52 pessoas doentes por surto alimentar, onde em 2007, foi relatado 41,3\% (1046) destes casos em 8 surtos alimentares (Tabela 1). Vale destacar ainda que esta média é elevada por conta da pequena quantidade de surtos identificados ao longo dos anos no Maranhão e da grande quantidade de pessoas acometidas por doenças veiculadas por alimentos.

As residências ( $\mathrm{n}=18 ; 37,5 \%)$ foram os locais de maior ocorrência de surtos alimentares para o período, no entanto não se faz a distinção entre a localização dos surtos (área rural ou urbana). Outros locais que merecem destaque são os casos dispersos em bairros $(6 ; 12,5 \%)$ e em restaurantes ou padarias $(5 ; 10,42 \%)$, como está representado na Tabela 2.

De acordo com Silva et al. (2017), a ocorrência de surtos alimentares em residências normalmente é elevada pela falta de conhecimento das boas práticas de preparação dos alimentos e falta de cuidado na manipulação. Além disso, não é difícil encontrar animais de estimação em ambientes de preparação de alimentos nas residências. Corroborando com os dados para o estado do Maranhão, Chang (2008) relatou 14 surtos alimentares em domicílios para o ano de 2005 em Recife-PE.

Também foi constatado 3 surtos alimentares em hospitais e/ou Unidades de Saúde no estado do Maranhão (ocorridos em 2007, 2010 e 2018). É importante citar que, no ambiente hospitalar, tem-se como parte dos cuidados com os pacientes internados, o fornecimento de uma alimentação com qualidade (Coloço, Holanda \& Portero-Mclellan, 2009), objetivando a manutenção da saúde dos indivíduos, já que muitas vezes estão imunodeprimidos.

Porém, os surtos alimentares nestes ambientes têm efeitos que vão além do impacto na saúde e prognóstico destes pacientes, acarretando normalmente em prejuízo financeiro (Zurlini, Lupino \& Nery, 2018), e estes achados demonstram a necessidade de mais atenção no estado do Maranhão.

Tabela 2. Estabelecimentos de ocorrência do surto no período de 2007 a 2019, no estado do Maranhão, 2020.

\begin{tabular}{cccc}
\hline & $\begin{array}{c}\text { Estabelecimentos/Local de } \\
\text { ocorrência do surto }\end{array}$ & $\mathbf{N}$ & $\%$ \\
\hline $\begin{array}{c}\text { Outras Instituições } \\
\text { (alojamento, trabalho) }\end{array}$ & 4 & 8,33 \\
Restaurantes/Padarias & 5 & 10,42 \\
(similares) & & \\
MA Hospital/Unidade de Saúde & 3 & 6,25 \\
& Casos dispersos no bairro & 6 & 12,50 \\
Casos dispersos pelo & 3 & 6,25 \\
município & & \\
Residência & 18 & 37,50 \\
Creche/Escola & 2 & 4,167 \\
Eventos & 3 & 6,25 \\
Outros & 4 & 8,33 \\
TOTAL & 48 & 100 \\
\hline
\end{tabular}

Fonte: Autores.

Quanto aos microrganismos envolvidos no estudo durante os anos de 2007 a 2019 no Maranhão,81,25\% dos casos não foi possível identificar o agente etiológico envolvido, normalmente um surto com agente não identificado ocorre pela notificação tardia, testes laboratoriais inadequados ou a falta de coleta de amostras clínicas e de alimentos (Figueiredo et al., 
2013). Como existe diversas variáveis que podem comprometer a segurança do alimento, muitas relacionadas com as características do patógeno envolvido, a identificação do agente etiológico é uma etapa fundamental para a elucidação do surto alimentar.

Dos casos em que foi possível a identificação do microrganismo, 6,25\% correspondeu a Salmonella spp., além de E. coli e rotavírus ambos com $4,17 \%$ e Staphylococcus aureus e coliformes com 2,08\% (Tabela 3).

Tabela 3. Alimentos causadores e agentes etiológicos envolvidos em surtos alimentares, no período de 2007 a 2019 , no estado do Maranhão - Brasil, 2020.

\begin{tabular}{|c|c|c|}
\hline Alimento Causador do surto & $\mathbf{N}$ & $\%$ \\
\hline Ovos e produtos à base de ovos & 2 & 4,17 \\
\hline Alimentos mistos & 6 & 12,50 \\
\hline Água & 7 & 14,58 \\
\hline Leite e derivados & 2 & 4,17 \\
\hline Doces e sobremesas & 1 & 2,08 \\
\hline $\begin{array}{c}\text { Frutas, produtos de frutas e } \\
\text { similares }\end{array}$ & 3 & 6,25 \\
\hline Múltiplos alimentos & 3 & 6,25 \\
\hline $\begin{array}{l}\text { Especiarias, temperos secos, } \\
\text { condimentos, molhos } \\
\text { industrializados e similares }\end{array}$ & 1 & 2,08 \\
\hline $\begin{array}{c}\text { Pescados, frutos do mar e } \\
\text { processados }\end{array}$ & 1 & 2,08 \\
\hline Ignorado & 21 & 43,75 \\
\hline Inconclusivo & 1 & 2,08 \\
\hline Agente Etiológico & $\mathbf{N}$ & $\%$ \\
\hline Salmonella sp. & 3 & 6,25 \\
\hline Escherichia coli & 2 & 4,17 \\
\hline Staphylococcus aureus & 1 & 2,08 \\
\hline Coliformes & 1 & 2,08 \\
\hline Rotavírus & 2 & 4,167 \\
\hline Ignorado & 39 & 81,25 \\
\hline TOTAL & 48 & 100 \\
\hline
\end{tabular}

Fonte: Autores.

A Salmonella spp. é um dos principais patógenos envolvidos em casos de Doenças veiculadas por Alimentos (Rodrigues, 2016), e é comumente relatada em surtos alimentares, especialmente em produtos de origem animal. Em uma pesquisa sobre surtos notificados no período de janeiro de 2009 a julho de 2014, no estado do Paraná, dos 105 surtos de doenças veiculadas por alimentos, 19 casos foram diagnosticados como sendo causados por contaminação por Salmonella spp. (Gabaron, Otutumi \& Júnior, 2015).

Apesar dos dados disponíveis no SINAN, a literatura apresenta um único estudo epidemiológico sobre surto por Salmonella spp. no Maranhão, em 1996 em São Luís, onde foi relacionado à Salmonella enteritidis envolvendo 11 pessoas após ingestão de pavê de maracujá contendo ovo cru (Alves et al., 2001).

No período estudado, ocorreram 15 óbitos, todos ocasionados por rotavírus em dois surtos alimentares (2007 e 2008 ). O rotavírus é visto como um importante agente etiológico de gastrenterite não bacteriana em muitas espécies de mamíferos e aves, sendo um grande causador de infecções graves em crianças menores de 5 anos (Parashar et al., 2003; Peixoto, 2013). O 
estado do Piauí também já relatou óbitos por rotavírus no município de Bom Jesus (Araújo et al., 2010).

Quanto ao tipo de alimento implicado, no presente estudo foi demonstrado que, do total de 48 surtos notificados, $43,75 \%$ tiveram o alimento causador ignorado e 2,08\% inconclusivo (Tabela 3). Vale ressaltar que é de extrema importância essa investigação, pois dessa forma se torna possível eliminar as fontes de contaminação, controlar e prevenir outros casos, além de poder melhorar a qualidade e segurança desse alimento ou água (Dive, 2006).

Dos $54,17 \%$ que foi possível identificar a origem, a água foi relatada em 7 surtos alimentares, demonstrando a necessidade de investimentos no sistema de abastecimento do estado do Maranhão. É importante citar, que recentemente ocorreu um surto através da ingestão de pescados e alimentos de origem marinha, fato importante por serem considerada uma das principais fontes de alimento da população maranhense, por outro lado, estima-se que esteja subnotificado, pois já existem vários achados de microrganismos patogênicos em pescados comercializados no Maranhão (Machado et al., 2020; Santos et al., 2019; Castro, Carvalho \& Costa, 2018).

As confirmações dos casos de surtos de DTA no presente estudo foram baseadas 52,08\% em achados clínicoepidemiológicos; enquanto que 14,58\% foram confirmados apenas por análises bromatológicas e 10,42\% por critérios clínicos somente. Porém, um número significativo de surtos ainda é incluído como inconclusivos e ignorados (22,92\%), como demonstrado na Tabela 4. Nem sempre os estudos clínico-epidemiológicos são responsáveis pela maioria das confirmações; o Governo do Estado do Paraná (2011) aponta que apenas 29,25\% dos surtos de DTA foram confirmados por este critério.

Tabela 4. Critérios utilizados para a confirmação do surto no período de 2007 a 2019 no Maranhão.

\begin{tabular}{ccr}
\hline Critério de confirmação & N & \% \\
\hline Clínico-epidemiológico & 25 & 52,08 \\
Laboratorial Clínico- & & \\
Bromatológico & 1 & 2,08 \\
Laboratorial Bromatológico & 6 & 12,50 \\
Laboratorial Clínico & 5 & 10,42 \\
Inconclusivo & & \\
Ignorado & 6 & 12,50 \\
TOTAL & 5 & 10,42 \\
& 48 & 100 \\
\hline
\end{tabular}

Fonte: Autores.

Essas diferenças encontradas normalmente estão conectadas com estrutura dos sistemas de vigilância, quando mais eficiente o trabalho dos órgãos competentes, mais rapidamente serão tomadas as decisões de instauração da investigação do surto, aumentando as chances de se encontrar amostras para complementar o inquérito.

Vale ressaltar que, de maneira preventiva, a vigilância sanitária promove a fiscalização dos locais que produzem, transportem e comercializam alimentos com a finalidade de proporcionar a boa prática na produção e manipulação de alimentos para que assim possa reduzir ou eliminar os possíveis riscos que a manipulação ou produção inadequada desses serviços e produtos podem causar ao consumidor (Sirtoli \& Comarella, 2018). Além disso, também é responsável pelo atendimento a denúncias, fiscalização para liberação de licença sanitária, ações programadas, investigação de surtos alimentares, coleta de alimentos e atividades educativas (Oliveira \& Cruz, 2015).

\section{Conclusão}

Conclui-se que as diferenças na quantidade de surtos notificados no período do estudo podem decorrer de uma 
possível subnotificação ocasionando lacunas nos resultados encontrados.

Além disso, o fato da maioria dos surtos investigados ter ocorrido em residências faz com que sejam necessárias medidas educativas para a população, sobretudo relativas aos cuidados com a manipulação dos alimentos, assim como de orientações para que as DTA, mesmo aquelas que possuem sintomas brandos, possam ser comunicadas aos serviços públicos, pois estes registros podem ser utilizados como subsídios para a realização de estudos epidemiológicos e elaboração de políticas públicas.

Novos estudos evidenciando a distribuição espaço-temporal dos surtos ocasionados podem revelar características epidemiológicas importantes (correlação entre IDH e saneamento com os surtos alimentares) para um melhor dimensionamento total das doenças veiculadas por alimentos e seus impactos na saúde da população do estado do Maranhão e na região nordeste.

\section{Referências}

Alves, L. M. C., Costa, F. N., Silva, M. I., Sales, S. S., \& Correa, M. R. (2001). Toxinfecçäo alimentar por Salmonella enteritidis: relato de um surto ocorrido em Säo Luís - MA. Revista Higiene Alimentar, 15(80/81),57-8.

Araújo, T. M. E., Dantas, J. M., Carvalho, C. E. F., \& Costa, M. A. O. (2010). Surto de diarreia por rotavírus no município de Bom Jesus (PI). Ciência e saúde coletiva, 15(1), 1039-1046.

Brasil. Ministério da Saúde. (2018). Surtos de doenças transmitidas por alimentos no Brasil. https://portalarquivos2.saude.gov.br/images/pdf/2018/julho/02/Apresentacao-Surtos-DTA-Junho-2018.pdf.

Brasil. Ministério da Saúde. Secretaria de Vigilância em Saúde. Departamento de Vigilância Epidemiológica. (2010). Manual integrado de vigilância, prevenção e controle de doenças transmitidas por alimentos. Brasília: Editora do Ministério da Saúde.

Castro, I. F. C., Carvalho, I. A., \& Costa, F. N. (2018). Pesquisa de Listeria monocytogenes e Vibrio parahaemolyticus em amostras de pescada amarela (Cynoscion acoupa) comercializadas na cidade de São Luís, MA. Revista Higiene Alimentar, 32, 99-102.

Chang, K. (2008). Surtos de doenças transmitidas por alimentos. Recife, 2005. Monografia (Especialização em Saúde Coletiva) - Residência Multiprofissional em Saúde Coletiva, Centro de Pesquisas Aggeu Magalhães, Fundação Oswaldo Cruz, Recife.

Coloço, R. B., Holanda, L. B. \& Portero-McLellan, K. C. (2009). Determinantes do grau de satisfação de pacientes internados referente a refeições oferecidas em um hospital universitário. Revista de Ciências Médicas, 10(3), 121-130.

Correia, C. B., Cunha, I. C., Coelho, A., Maia, C., Pena, C., Bonito, C. C., Flores, C., Moura, I. B., Sousa, I., Barreira, M. J., Toscano, M. M., Furtado, R., Marcos, S., Santos, S., Lopes, T. T., Saraiva, M., \& Castanheira, I. (2019). Investigação laboratorial de surtos de toxinfeção alimentar: dados referentes a 2017. Boletim Epidemiológico, 25(3), 13-19.

Dive - Diretoria de Vigilância Epidemiológica. (2006). Manual de orientação para investição em surtos de DTA. http://www.dive.sc.gov.br/conteudos/publicacoes/manuais_cartilhas/Manual_de_Orientacao_para_Investigacao_em_Surtos_de_DTA.pdf.

Figueiredo, D., Tim, L. N., Cecconi, M. C. P., Both, J. M. C., Soeiro, M. L. T., Ramos, R. C., Haas, S., \& Longaray, S. M. (2013). Programa de Vigilância Epidemiológica das Doenças de Transmissão Hídricas e Alimentares - VE-DTHA. Secretaria Estadual da Saúde. Boletim Epidemiológico, 15(3), 1-8.

Gabaron, D. A., Otutumi, L. K., \& Júnior R. P. (2015). Surtos de salmonelose notificados no período de janeiro de 2009 a julho de 2014 no estado do Paraná, Brasil. Arquivos de Ciências Veterinárias e Zoologia da UNIPAR, 18(1), 33-37.

Governo do Estado do Paraná. Secretaria de Estado da Saúde do Paraná. (2011). Surto alimentar. http://www.saude.pr.gov.br/CSA/Surto_alimentar/index.htm.

Guilherme D. L., \& Esteves, D. C. (2017). Doenças transmitidas por alimentos e água. Conexão Eletrônica, 14(1), 390-401.

Machado, E. F., Lopes, H. S., Pereira, D. M., Leoncio, G. G., Pereira, L. E. C., Queiroz, M. L. M., \& Costa, F. N. (2020). Alterações sensoriais, microbiológicas e químicas da pescada amarela (Cynoscionacoupa) e do peixe-serra (Scomberomorus brasiliensis) desembarcados em portos no Maranhão. Brazilian Journal of Development, 6, 26662-26676.

Melo, E. S., Amorim, W. R., Pinheiro, R. E. E., Corrêa, P. G. N., Carvalho, S. M. R., Santos, A. R. S. S., Barros, D. S., Alencar, E. T., Oliveira, C., Mendes, C. A., \& Sousa, F. V. (2018). Doenças transmitidas por alimentos e principais agentes bacterianos envolvidos em surtos no Brasil: revisão. PubVet, 12(10), 19 .

Metodologia da pesquisa científica. UAB/NTE/UFSM. https://repositorio.ufsm.br/bitstream/handle/1/15824/Lic_Computacao_Metodologia-PesquisaCientifica.pdf?sequence $=1$.

Oliveira, C. M., \& Cruz, M. M. (2015). Sistema de Vigilância em Saúde no Brasil: avanços e desafios. Saúde Debate, 39(104), $255-267$. 
Research, Society and Development, v. 10, n. 1, e36010111728, 2021 (CC BY 4.0) | ISSN 2525-3409 | DOI: http://dx.doi.org/10.33448/rsd-v10i1.11728

Parashar, U. D., Hummelman, E. G., Bresee, J. S., Miller, M. A., Glass. \& R. I. (2003). Global Illness and Deaths Caused by Rotavirus Disease in Children. Emerging Infectious Diseases, 9(5), 565-572.

Peixoto, I. B. (2013). Estudo e caracterização molecular do rotavírus humano na cidade de Salvador (BA). Dissertação (Mestrado em Biotecnologia) Programa de Pesquisa e Pós- Graduação em Biotecnologia, Instituto de Ciência e Saúde, Universidade Federal da Bahia, Salvador.

Rodrigues, C. F. (2016). Pesquisa de coliformes e Salmonella spp. em ovos comercializados em feira livre, no município de Espigão do Oeste - Rondônia. Dissertação (Mestrado), São Paulo.

Santos, E. J. R., Galeno, L. S., Bastos, L. S., Carvalho, I. A., \& Costa, F. N. (2019). Qualidade higiênico-sanitária de tambaqui (Colossoma macropomum) comercializado na cidade de São Luís - MA. Ciência Animal Brasileira, 20, 1-12.

Silva, J. C. G., Filho, M. M. S., Nascimento, G. V., Pereira, D. A. B., \& Costa-Junior, C. E. O. (2017) Incidência de doenças transmitidas por alimentos (DTA) no estado de Pernambuco, um acompanhamento dos dados epidemiológicos nos últimos anos. Ciências Biológicas e de Saúde Unit, 3(1), 23-34.

Sirtoli, D. B., \& Comarella, L. (2018). O papel da vigilância sanitária na prevenção das doenças transmitidas por alimentos (DTA). Revista Saúde e Desenvolvimento, 12(10), 197-209.

WHO - World Health Organization. (2019). World health statistics 2019: monitoring health for the SDGs, sustainable development goals. Geneva: World Health Organization.

Zurlini, A. C., Lupino, C. S., Nery, J. S. C., \& Santos, M. C. H. G. (2018). Evaluation of the hygienico sanitary control of food production in food and hospital nutritionunits. Revista Higiene Alimentar, 32(284/285), 51-55. 